\title{
Laser Holographic Approaches for Rapid Bacterial Species Identification
} YongKeun Park*

Department of Physics, Korea Advanced Institute of Science and Technology, Daejeon 305-701, Republic of Korea

Holographic techniques with machine learning algorithms can enable rapid identification of bacterial species bypassing timeconsuming culture and preparation processes.

Bacterial infections are one of the major sources of illnesses and diseases and thus pose serious problems in clinics, hospitals, and food industries. Bacterial poisoning often has severe implications such as pneumonia, sepsis, meningitis, and gastroenteritis, which can be lethal unless immediate and appropriate treatment is given. Unfortunately, the present gold standard techniques for bacterial identification are generally not fast enough to meet the clinical demands for appropriate patient care. For example, bacterial blood culture followed by susceptibility testing for drug resistance requires days for preliminary results and is often negative for fulminant cases. Real-time quantitative polymerase chain reaction ( $\mathrm{qPCR}$ ) followed by gene sequencing is faster and more robust; however, it still requires hours and is often too expensive for resource-limited environments.

Traditionally, bright-field microscopy has been widely used for imaging individual bacteria. However, bacterial species can be difficult to identify using conventional microscopic techniques because they only provide limited morphological information about individual bacterium such as length, width, sphericity, etc., and most bacterial species share similar morphological features (Figure 1a). While immunofluorescence labeling techniques can provide highly molecularspecific information, they require expensive and time-consuming labeling processes. Recently, non-invasive optical spectroscopic methods have been proposed for this purpose, including Fourier transform infrared spectroscopy (FTIR) [1] and Raman spectroscopy [2]. Although these spectroscopic methods simplified sample processes in comparison to conventional biochemical approaches, the speed of measurements is still limited due to the culture process or weak signal.

Alternatively, holographic techniques have been proposed for rapid and effective species identification. Transparent microscopic objects such as individual bacteria provide very poor imaging contrast in light absorption. However, refractive index distributions inside individual bacteria result in excellent imaging contrast in optical phase images. When employing optical holographic techniques, these optical phase images can be quantitatively and precisely obtained (Figure 1b). Furthermore, quantitative phase imaging helps to identify different species, as two different bacterial species with similar shapes can have different subcellular structures and protein compositions, resulting in distinct optical phase information.

Various holographic approaches have been introduced to address this issue in microbiology. Historically, angle-resolved light scattering patterns from bacterial suspensions and colonies have been extensively investigated [3-5]. Since light scattering patterns from objects are determined by the refractive index distributions of a sample, the angleresolved light scattering patterns of a sample provide a means of labelfree identification. Despite significant efforts, however, measuring light scattering patterns from individual bacterium, which is crucial for rapid bacterial identification without culturing, has remained challenging due to technical issues: light scattering signals from an individual bacterium are extremely small and the light scattering signal generally spans over all scattering directions with an extremely high dynamic range.

Recent advances in quantitative phase imaging techniques have renewed interest in using optical phase information for bacterial identification [6]. Precise measurements of the optical phase images of individual bacterium can be achieved using a stable and precise digital holographic recording. The Fourier-transform light scattering technique, a computation method to convert a measured holographic image to an angle-resolved light scattering pattern [7], was utilized to retrieve the light scattering pattern of a bacterium with unprecedented sensitivity and precision [8].

These techniques in combination with a pattern recognition algorithm, which is used for face recognition in surveillance, showed promise for rapid and effective identification of bacterial species [9]. Recently, our group collaborated to demonstrate a proof of principle. We examined four different bacterial species (Listeria monocytogenes, Escherichia coli, Lactobacillus casei, and Bacillus subtilis). These four rod-like bacteria have nearly identical appearance under a conventional bright-field microscope. The measurements of holographic light scattering patterns, however, enable them to be identified with accuracy greater than 94 percent. These holographic approaches can also be utilized to perform antimicrobial susceptibility tests and to analyze antimicrobial activities of several compounds [10].

Much work is still ahead to realize label-free bacterial identification that can be used as a rapid, cost-effective solution for hospitals and food industries worldwide. The sensitivity of the approach has to be further improved in order to identify similar bacterial species of the same genus. Other sensing modalities may need to be exploited to improve the sensitivity; for example, hyperspectral holographic imaging [11] or polarization sensitive measurements [12] can also be combined. Furthermore, systematic analysis about sample processing would be performed to utilize the holographic approach as a reference method, because sample conditions are important factors which can alter the diagnosis in clinical infectious diseases [13]. In addition, current setups for holographic measurements are bulky and require complicated alignments of light, which requires optical experts. Thus, simplified instrumentations [14] or mobile-phone-based approaches should be introduced for point-of-care solutions. In addition, high-throughput

*Corresponding author: YongKeun Park, Department of Physics, Korea Advanced Institute of Science and Technology, Daejeon 305-701, Republic of Korea, Tel: +82 42-350-2114; E-mail: yk.park@kaist.ac.kr

Received July 02, 2015; Accepted July 22, 2015; Published July 29, 2015

Citation: Park YK (2015) Laser Holographic Approaches for Rapid Bacterial Species Identification. J Microb Biochem Technol 7: 234-235. doi:10.4172/19485948.1000213

Copyright: (c) 2015 Park YK. This is an open-access article distributed under the terms of the Creative Commons Attribution License, which permits unrestricted use, distribution, and reproduction in any medium, provided the original author and source are credited. 
Citation: Park YK (2015) Laser Holographic Approaches for Rapid Bacterial Species Identification. J Microb Biochem Technol 7:4 234-235. doi:10.4172/1948-5948.1000213

a

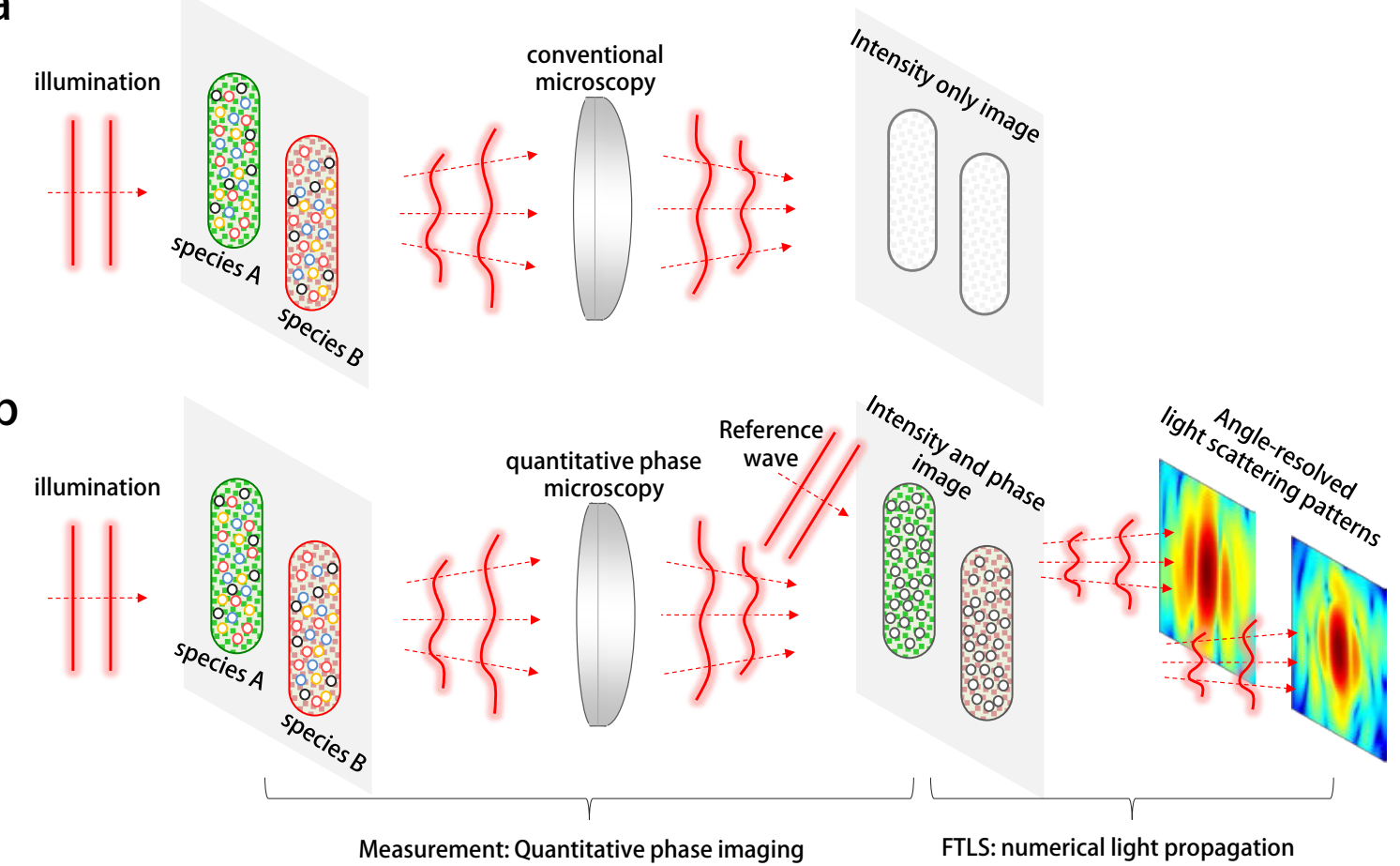

Figure 1: Microscopic imaging of individual bacteria for identification. (a) Conventional light microscopy only provides image contrast based on light absorption, which cannot distinguish different bacterial species. (b) Quantitative phase microscopy measures both intensity and phase images, which are sensitive to structures and compositions of bacterial components, and then numerically propagated using Fourier transform light scattering (FTLS) which can be utilized for bacterial species identification.

measurement schemes also can be introduced for clinical applications. Most importantly, however, recent updates to holographic techniques have provided novel approaches for rapid bacterial identification.

\section{References}

1. Preisner O, Lopes JA, Guiomar R, Machado J, Menezes JC (2007) Fourier transform infrared (FT-IR) spectroscopy in bacteriology: Towards a reference method for bacteria discrimination. Anal Bioanal Chem 387: 1739-1748.

2. Schuster KC, Reese I, Urlaub E, Gapes JR, Lendl B (2000) Multidimensional information on the chemical composition of single bacterial cells by confocal Raman micro spectroscopy. Analytical chemistry 72: 5529-5534.

3. Wyatt PJ (1969) Identification of bacteria by differential light scattering. Nature 221: $1257-1258$

4. Bae E, Bai N, Aroonnual A, Robinson JP, Bhunia AK, et al. (2010) Modeling light propagation through bacterial colonies and its correlation with forward scattering patterns. J Biomed Opt 15: 045001.

5. Suchwalko A, Buzalewicz I, Wieliczko A, Podbielska H (2013) Bacteria species identification by the statistical analysis of bacterial colonies Fresnel patterns. Opt Express 21: 11322-11337.

6. Lee K, Kim K, Jung J, Heo J, Cho S, et al. (2013) Quantitative phase imaging techniques for the study of cell pathophysiology: From principles to applications. Sensors (Basel) 13: 4170-4191.

7. Ding H, Wang Z, Nguyen F, Boppart SA, Popescu G (2008) Fourier transform light scattering of inhomogeneous and dynamic structures. Phys Rev Lett 101 238102.

8. Jo Y, Jung J, Lee JW, Shin D, Park H, et al. (2014) Angle-resolved light scattering of individual rod-shaped bacteria based on Fourier transform light scattering. Sci Rep 4: 5090.

9. Jo Y, Jung J, Kim MH, Park H, Kang SJ, et al. (2015) Label-free identification of individual bacteria using Fourier transform light scattering. Optics Express 23: $15792-15805$.
10. Usman MS, El Zowalaty ME, Shameli K, Zainuddin N, Salama M, et al. (2013) Synthesis, characterization, and antimicrobial properties of copper nanoparticles. Int J Nanomedicine 8: 4467-4479.

11. Jung J, Kim K, Yu H, Lee K, Lee S, et al. (2014) Biomedical applications of holographic micro spectroscopy [invited]. Appl Opt 53: G111-122.

12. Kim Y, Jeong J, Jang J, Kim MW, Park Y (2012) Polarization holographic microscopy for extracting spatio-temporally resolved Jones matrix. Opt Express 20: 9948-9955.

13. Maquelin K, Kirschner C, Choo-Smith LP, Ngo-Thi N, Van Vreeswijk T, et al. (2003) Prospective study of the performance of vibrational spectroscopies for rapid identification of bacterial and fungal pathogens recovered from blood cultures. Journal of Clinical Microbiology 41: 324-329.

14. Lee K, Park Y (2014) Quantitative phase imaging unit. Opt Lett 39: 3630-3633. 